\title{
Umbilical cord clamping and skin-to-skin contact in deliveries from women positive for COVID-19
}

\author{
Inmaculada Mejía ${ }^{1}$, Rita Salvador López ${ }^{2}$, Emérita García Rosas ${ }^{3}$, Iria Rodriguez de la \\ Torre $^{1}$, José Montes García ${ }^{4}$, and Oscar Martinez-Perez ${ }^{5}$ \\ ${ }^{1}$ Hospital Universitario 12 de Octubre \\ ${ }^{2}$ Hospital Universitario Puerta del Hierro Majadahonda \\ ${ }^{3}$ Hospital del Mar \\ ${ }^{4}$ Effice \\ ${ }^{5}$ Puerta del Hierro University Hospital of Majadahonda
}

July 28, 2020

\begin{abstract}
OBJECTIVE: To demonstrate that delayed cord clamping (DCC) is safe in mothers with confirmed SARS-CoV-2 infection. DESIGN, SETTING, AND PARTICIPANTS: Prospective, observational study involving epidemiological information from 403 pregnant women with SARS-CoV-2 between March 1st and May 31st, 2020. Data were collected from 70 centers that participate in the Spanish Registry of COVID-19. MAIN OUTCOMES AND MEASURES: The rate of perinatal transmission of SARSCoV-2 and development of COVID-19 disease in neonates at day 14 of the delivery. RESULTS: The Early cord clamping (ECC) group consisted of 231 infants (57.3\%), whereas the DCC group consisted of 172 infants (42.7\%). A total of 5 positive cases $(1.7 \%$ of total tests performed) were identified with the nasopharyngeal PCR tests, 2 from the ECC group (1.7\%) and 3 from the DCC group (3.6\%). No significant differences between groups were found regarding neonatal tests for COVID-19. No confirmed cases of vertical transmission were detected. The percentage of mothers who made skin-to-skin contact within the first 24 hours after delivery was significantly higher in the DCC group (84.3\% versus $45.9 \%)$. Breastfeeding in the immediate postpartum period was also significantly higher in the DCC group ( $77.3 \%$ versus $50.2 \%)$. CONCLUSIONS: The results of our study have been similar to early cord clamping practices, no skin-to-skin contact, and suppression or delay of breastfeeding. FUNDING: This study was fully funded with public funds from the Institute of Health Carlos III and co-financed with FEDER funds. KEYWORDS: COVID-19, umbilical cord clamping, skin-to-skin, breastfeeding, vertical transmission, safety.
\end{abstract}

\section{Introduction}

On January $12^{\text {th }}, 2020$ Chinese Authorities shared the genetic sequence of a novel type of virus belonging to the Coronaviridae family, given the name severe acute respiratory syndrome coronavirus 2 (SARS-CoV2). ${ }^{1}$ By international consensus, its related disease has been called coronavirus disease 2019 (COVID-19). The World Health Organization (WHO) declared COVID-19 a pandemic on $11^{\text {th }}$ March due to the prevalence, spread, and severity of the disease. ${ }^{2}$ To date, a higher predisposition to infection of pregnant women compared to the general population has not been proven; however, evidence suggests they have a greater susceptibility to develop pneumonia. ${ }^{3}$ Moreover, the clinical course seems more severe among them. Higher rates of preterm births and cesarean deliveries have also been detected; the latter being associated with an elevated risk of clinical impairment. ${ }^{4}$ Protocols for isolation and social distancing in pregnant women are the same as those for the general population. ${ }^{5}$ There is no strong evidence supporting the existence of vertical transmission. ${ }^{6-8}$ Some case reports suggest the possible transplacental transmission of SARS-CoV-2. ${ }^{9}$ Nevertheless, although vertical transmission has been described, it is very uncommon. Certain practices 
during vaginal and cesarean deliveries have been modified during the pandemic. Some centers have suppressed or substantially minimized delayed cord clamping (DCC), mother/infant skin-to-skin contact, and breastfeeding. ${ }^{8,10}$ However, $\mathrm{WHO}^{11}$ and diverse Scientific Societies (Centers for Disease Control and Prevention, CDC; ${ }^{12}$ The American College of Obstetricians and Gynecologists, ACOG, ${ }^{13}$ National Institute for Health and Care Excellence, NICE; ${ }^{14}$ Spanish Society of Obstetrics and Gynecology, SEGO; ${ }^{5}$ or The Spanish Neonatology Society, SENEO $;^{5}$ among others) recommend these practices in COVID-19 positive mothers because the benefits outweigh the risks and the likelihood of neonatal infection is actually very low. The objective of the present study was to demonstrate that DCC is safe in mothers with confirmed SARS-CoV-2 infection.

\section{Materials and Methods}

\section{Study design and population}

This prospective, observational study involved epidemiological information from pregnant women with SARSCoV-2 between March $1^{\text {st }}$ and May $31^{\text {st }}, 2020$. Data were collected from the Spanish Registry of COVID$19 .{ }^{15}$ A total of 100 Spanish centers participate in the Registry, representing $49.95 \%(\mathrm{n}=172,000)$ of total deliveries $(\mathrm{N}=359,770)$ carried out in 2019 in Spain. ${ }^{16}$ Finally a total of 70 centers included cases in the present study. The study was firstly approved by the Puerta de Hierro University Hospital Ethics Committee, and subsequently by the Ethics Committee of each participating hospital. Procedures were in concordance with the Declaration of Helsinki. Oral informed consent was obtained from each participant.

\section{Analyzed variables}

Women were differentiated according to the timing of cord clamping (early or delayed). Early cord clamping (ECC) and DCC were established when performed $<30$ or $>30$ seconds after the delivery, respectively. Primary variables included: the rate of perinatal transmission of SARS-CoV-2 and development of COVID19 disease in neonates at day 14 of the delivery. Perinatal transmission was defined by a positive PCR in a nasopharyngeal sample from the neonate. Given the lack of a uniform criterion about neonatal infection, the diagnosis was made by PCR from a nasopharyngeal sample, following specific considerations. If the PCR was positive within 12 hours after delivery, it was repeated. If this second PCR was negative, the first PCR was then considered as contaminated or a false positive; however, if positive, the infection was corroborated. Each case was followed-up at 14 days after delivery, by phone. The state of health of each neonate was confirmed during the writing of this manuscript (June 2020). Secondary variables included: the need for neonatal resuscitation, admission at the intensive care unit (ICU), neonatal symptomatology suggestive of COVID-19, and rates of skin-to-skin contact and early breastfeeding. Neonatal symptoms were evaluated at day 14 after delivery, by completing a clinical questionnaire during a phone interview.

\section{Statistical analysis}

For the descriptive analysis, categorical variables were expressed as absolute and relative frequencies; and quantitative values as mean and range (minimum-maximum values). Comparisons between ECC and DCC groups were carried out with the chi-square or Fisher Exact tests for categorical variables, and t-test or U Mann-Whitney test for quantitative ones, when appropriate. Statistical significance was established with $P$ $<.05$. All statistical analyses were performed with SAS 9.4.

\section{Results}

Data from 475 pregnant women with confirmed SARS-CoV-2 infection and their deliveries were initially included in the study; however, 72 were discarded out because of a lack of information about the timing of the cord clamping. Therefore, 403 cases were finally analyzed. ECC was performed on 231 neonates (57.3\%), whereas $172(42.7 \%)$ received DCC. No significant differences were found between ECC and DCC groups in maternal age and time between COVID-19 diagnosis and delivery (Table 1). Regarding maternal symptomatology at the time of delivery, $82(35.5 \%)$ and $149(64.5 \%)$ women showed COVID-19 symptoms or were asymptomatic in the ECC group, respectively. In the case of DCC, 30 women (17.4\%) showed symptoms, whereas $142(82.6 \%)$ were asymptomatic. The gestational age at delivery with ECC was significantly lower 
than DCC $(37+9$ versus $38+8$ weeks, $P=.001)$. The number of instrumental and cesarean deliveries were higher with ECC than DCC (13.0\% versus $8.1 \%$ for instrumental ones, and $45.9 \%$ versus $17.4 \%$ for cesarean); whereas the number of eutocic deliveries was higher for DCC (74.4\% versus $41.1 \%)$. The weight at birth was significantly higher with DCC than ECC (3,210.4 versus 3,065.7 grams, $P=.037)$. Although statistically significant, this difference was not clinically relevant.

A total of 5 positive cases (1.7\% of total tests performed) were identified with the nasopharyngeal PCR tests, specifically 2 from the ECC $(1.7 \%)$ and 3 from the DCC group (3.6\%; Table 2). No significant differences between groups were found regarding neonatal tests for COVID-19 $(P=.390)$. All positive cases reported within 12 hours after delivery resulted negative in the confirmation test performed between 12 and 48 hours post-delivery. Therefore, no confirmed cases of vertical transmission were detected. A new positive case was found within 12-48 hours of delivery, which was possibly related with horizontal transmission, through contact with a relative without the use of protection measures (and unknown infection). None of the neonates experienced COVID-19 at day 14 after delivery.

The percentage of mothers who made skin-to-skin contact within the first 24 hours after delivery was significantly higher with DCC ( $84.3 \%$ versus $45.9 \%, P=.001)$. Breastfeeding in the immediate postpartum period was also significantly higher with DCC than ECC $(77.3 \%$ versus $50.2 \%, P=.001)$.

No significant differences between groups were found regarding arterial $\mathrm{pH}$ and Apgar score at 5 minutes in neonates. A higher percentage of admissions to the ICU were reported in the ECC $(16.5 \%$ versus $8.1 \%, P=$ $.015)$.

Considering the temporal distribution, ECC was more prevalent than DCC during the first few days of the pandemic (5.2\% versus $2.3 \%$ between $1^{\text {st }}$ and $15^{\text {th }}$ March, $25.5 \%$ versus $15.1 \%$ between $16^{\text {th }}$ and $31^{\text {st }}$ March, and $31.6 \%$ versus $20.9 \%$ between $1^{\text {st }}$ and $15^{\text {th }}$ April. Time evolution is shown in Table 3.) The main reason for an ECC was due to maternal COVID-19 disease (37.2\%)

\section{Discussion}

Our study supports the recommendations from WHO, ${ }^{11} \mathrm{CDC},{ }^{12}$ and the Spanish Government ${ }^{5}$ on the management of deliveries and neonate care during the COVID-19 pandemic. Current evidence does not conclusively support intrauterine transmission of SARS-CoV-2.$^{6-8}$ However it is known that DCC, and not ECC, can reduce the risk of death before hospital discharge in preterm neonates, ${ }^{17}$ and provide benefits in those born at term. Thus, there is no evidence for not continuing to perform it. The routine separation of the neonate from the mother interferes in the mother/infant relationship. ${ }^{18} \mathrm{~A}$ woman with a probable or confirmed suspicion of COVID-19 disease can give skin-to-skin contact in the delivery room, and exclusively breastfeed her baby. Breastfeeding improves the health of both mother and infant, results in benefits for the families, and has a positive social and economic impact. ${ }^{18}$ On the whole, this current pandemic has led to combining the promotion of breastfeeding with adequate measures of infection control (wearing a mask, frequent hand washing, and social distancing). In Spain, the lack of solid evidence on the vertical transmission of the coronavirus during the initial days of the pandemic led to very conservative recommendations from the Spanish Ministry of Health for the management of deliveries in women with COVID-19. ${ }^{5}$ ECC, little skin-to-skin contact, and negativity to breastfeeding practices, were the decisions made in many cases. According to our study, ECC was more prevalent over DCC during the early period. Both ECC and DCC were equally used between $16^{\text {th }}$ and $30^{\text {th }}$ April. Once Healthcare Authorities proclaimed the safety of these interventions, ${ }^{19}$ the clinical practice took a new stance and progressively returned to DCC and early skin-to-skin contact. Moreover, hygienic measures (wearing a mask and frequent hand washing) were introduced to avoid mother/infant transmission during breastfeeding.

The characterization of our present study is the provision of perinatal outcomes of neonates born to COVID19-positive mothers with DCC, practicing skin-to-skin contact and early breastfeeding under appropriate safety measures. Moreover, we included perinatal outcomes of neonates with ECC due to diverse reasons. No significant differences in COVID-19 infections were detected between the ECC and the DCC groups. Likewise, no COVID-19 symptomatology was found in neonates at day 14 of follow-up in both groups. This 
fact corroborates the safety of DCC and skin-to-skin contact and breastfeeding practices in women with COVID-19, in agreement with main Scientific Societies. ${ }^{5,11,12}$ It is interesting to highlight in our study the large percentage of preterm neonates with ECC. The fear of vertical transmission of COVID-19 (principle reason for choosing ECC) probably caused the decrease in the number of DCC in these neonates, who in turn are those who may benefit most from this intervention.

The possible intrapartum infection of neonates has been described. ${ }^{19}$ The suspicion could originate from a positive nasopharyngeal PRC test within 12 hours after delivery and confirmed within 24-48 hours. Horizontal transmission is suspected in the case of a positive nasopharyngeal PRC test within 24-48 hours, but a prior negative one. ${ }^{20}$ In our study, we reported 5 cases of positive nasopharyngeal PRC within 12 hours of delivery, and all were negative in the confirmation test within 24-48 hours post-delivery. This result points to the probable contamination during sample collection or a false positive. Another study from our research group also evidenced two positive PCR cases (cesarean deliveries at term), who experienced COVID-19 symptoms within 10 days of delivery. ${ }^{4}$ In that study, the initial test was negative but positive when confirmed. In both cases, neonates were in contact with parents immediately after delivery. The COVID-19 symptoms resolved within 48 hours. No information about the timing of the cord clamping was available. In our present study, we reported one positive case within 12-48 hours after delivery, possibly related to contact with a relative unaware of being infected. This neonate showed COVID-19 symptoms for some days and did not require admission into the ICU. None of the neonates from our cohort showed COVID-19 symptoms when the phone evaluation took place at day 14 after delivery, and while writing this manuscript (June 2020). Moreover, none of the neonates required admission into the ICU due to severe symptomatology of SARS-CoV-2 infection.

\section{Strengths and Limitations}

The main strength of the study is the number of registered cases (403 deliveries from 70 centers across Spain), being one of the largest cohorts described. Furthermore, it represents a novel topic because, to our knowledge, there are no studies that have analyzed perinatal outcomes in neonates born to mothers with COVID-19 regarding the timing of cordon clamping, or that have evaluated the safety of DCC, skin-to-skin contact, and breastfeeding practices in these neonates.

On the other hand, our study has several limitations. Routine serology tests (for determining the immunological state after delivery) were not performed in neonates born to mothers with confirmed COVID-19 due to the lack of availability at the beginning of the pandemic in Spain, and later, once they were available, due to the diversity of tests and protocols in the distinct centers. Furthermore, the clinical questionnaire (for evaluating the neonatal symptoms) was not normalized and homogeneous in all the centers. The determination of parts of the virus in the neonate with ultrasensitive tests does not mean the existence of the complete virus with infective capacity. We did not know if the healthcare professionals who assisted the deliveries or the relatives who visited the neonates were SARS-CoV-2 positive or not. We were able to trace this association in one of the positive PCR cases; nevertheless, in a pandemic like this, we do believe that the present results are unique and relevant because of the difficult circumstances in which they were obtained. In the future, once serologic diagnostic methods improve and become more available, there will be a need to determine the presence and evolution of immunoglobulins against SARS-CoV-2 in order to clarify whether transplacental transmission does exist. Further long-term, prospective studies with neonates are thus required.

\section{Conclusions}

Our study supports the approaches of delayed cord clamping, skin-to-skin contact, and early breastfeeding in mothers with COVID-19. Perinatal results associated to these practices have been similar to those with early cord clamping, no skin-to-skin contact, and suppression or delay of breastfeeding.

\section{Acknowledgements}

Authors would express gratitude to Meisys for assisting in the preparation of the manuscript.

\section{Disclosure Statement}


The authors report no conflict of interest.

\section{Contribution to Authorship}

All the authors certify that have participated sufficiently and equally in the work.

\section{Details of Ethics Approval}

The study was firstly approved by the Puerta de Hierro University Hospital Ethics Committee, and subsequently by the Ethics Committee of each participating hospital. Procedures were in concordance with the Declaration of Helsinki. Oral informed consent was obtained from each participant.

\section{Funding}

This study was fully funded with public funds obtained in competitive calls: grant COV20/00020 from the Institute of Health Carlos III and co-financed with FEDER funds.

\section{References}

1. World Health Organization. Emergencies preparedness, response. Novel Coronavirus - China. Accessed June 29, 2020. https://www.who.int/csr/don/12-january-2020-novel-coronavirus-china/en/

2. World Health Organization. Archived: WHO Timeline - COVID-19. Accessed June 29, 2020.https://www.who.int/news-room/detail/27-04-2020-who-timeline-covid-19

3. San-Juan R, Barbero P, Fernández-Ruiz M, López-Medrano F, Lizasoáin M, Hernández-Jiménez P, et al. Incidence and clinical profiles of COVID-19 pneumonia in pregnant women: A single-centre cohort study from Spain. EClinicalMedicine . 2020;23:100407. doi: 10.1016/j.eclinm.2020.100407

4. Martínez-Perez O, Vouga M, Cruz Melguizo S, Forcen Acebal L, Panchaud A, Muñoz-Chápuli M, et al. Association between mode of delivery among pregnant women with COVID-19 and maternal and neonatal outcomes in Spain. JAMA . 2020;e2010125. doi:10.1001/jama.2020.10125

5. Government of Spain. Ministry of Health. Manejo de la mujer embarazada y el recién nacido con COVID-19. Accessed June 29, 2020.https://www.mscbs.gob.es/en/profesionales/saludPublica/ccayes/alertasActual/nCov-

China/documentos/Documento_manejo_embarazo_recien_nacido.pdf

6. Karimi-Zarchi M, Neamatzadeh H, Dastgheib SA, Abbasi H, Mirjalili SR, Behforouz A, et al. Vertical transmission of coronavirus disease 19 (COVID-19) from infected pregnant mothers to neonates: A review.Fetal Pediatr Pathol. 2020;39(3):246-250. doi: 10.1080/15513815.2020.1747120

7. Liu W, Wang J, Li W, Zhou Z, Liu S, Rong Z. Clinical characteristics of 19 neonates born to mothers with COVID-19. Front Med.2020;14(2):193-198. doi: 10.1007/s11684-020-0772-y

8. Yang P, Wang X, Liu P, Wei C, He B, Zheng J, et al. Clinical characteristics and risk assessment of newborns born to mothers with COVID-19. J Clin Virol. 2020;127:104356. doi: 10.1016/j.jcv.2020.104356

9. Alzamora MC, Paredes T, Caceres D, Webb CM, Valdez LM, La Rosa M. Severe COVID-19 during pregnancy and possible vertical transmission. Am J Perinatol. 2020;37(8):861-865. doi: 10.1055/s-0040-1710050

10. Favre G, Pomar L, Qi X, Nielsen-Saines K, Musso D, Baud D. Guidelines for pregnant women with suspected SARS-CoV-2 infection. Lancet Infect Dis. 2020;20(6):652-653. doi: 10.1016/S1473-3099(20)30157-2

11. World Health Organization. Clinical management of COVID-19: Interim guidance, 27 May 2020. Accessed June 29, 2020.https://apps.who.int/iris/handle/10665/332196

12. Centers for Disease Control and Prevention. Coronavirus disease (COVID-19) and Breastfeeding. Accessed June 29, 2020.https://www.cdc.gov/breastfeeding/breastfeeding-special-circumstances/maternal-orinfant-illnesses/covid-19-and-breastfeeding.html 
13. The American College of Obstetricians and Gynecologists. COVID-19 FAQs for ObstetricianGynecologists, Obstetrics. Accessed June 29, 2020.https://www.acog.org/clinical-information/physicianfaqs/covid-19-faqs-for-ob-gyns-obstetrics

14. National Institute for Health and Care Excellence. Accessed June 29, 2020.https://www.rcog.org.uk/globalassets/documents/guidelines/2020-06-18-coronavirus-covid-19-

infection-in-pregnancy.pdf

15. Government of Spain. Instituto de Salud Carlos III. Epidemiological record of pregnant women with COVID-19. Accessed June 29, 2020.http://eu-isciii.es/covidfundinspain/epidemiological-record-of-pregnantwomen-with-covid-19/

16. Instituto Nacional de Estadística. Accessed June 29, 2020.https://www.ine.es/dyngs/INEbase/es/operacion.htm? $c=$ Estadistica_CÉcid=1254736177007\&̈menu=ultiDatos\&6idp=12547355

17. Rabe H, Gyte GM, Díaz-Rossello JL, Duley L. Effect of timing of umbilical cord clamping and other strategies to influence placental transfusion at preterm birth on maternal and infant outcomes. Cochrane Database Syst Rev. 2019;9(9):CD003248. doi:10.1002/14651858.CD003248.pub4.

18. World Health Organization. Implementation guidance 2018. Protecting, promoting and supporting Breastfeeding in facilities providing maternity and newborn services: the revised Baby-friendly hospital initiative. 2018th ed. 2018; 54p. Accessed June 29, 2020.http://apps.who.int/bookorders

19. Huntley BJF, Huntley ES, Di Mascio D, Chen T, Berghella V, Chauhan SP. Rates of maternal and perinatal mortality and vertical transmission in pregnancies complicated by severe acute respiratory syndrome coronavirus 2 (SARS-Co-V-2) infection: A systematic review. Obstet Gynecol. 2020 ;June 9. doi:10.1097/AOG.0000000000004010

20. Shah PS, Diambomba Y, Acharya G, Morris SK, Bitnun A. Classification system and case definition for SARS-CoV-2 infection in pregnant women, fetuses, and neonates. Acta Obstet Gynecol Scand.2020;99(5):565568. doi:10.1111/aogs.13870

\section{Hosted file}

Tabla 1.docx available at https://authorea.com/users/346819/articles/472667-umbilical-cordclamping-and-skin-to-skin-contact-in-deliveries-from-women-positive-for-covid-19

\section{Hosted file}

Tabla 2.docx available at https://authorea.com/users/346819/articles/472667-umbilical-cordclamping-and-skin-to-skin-contact-in-deliveries-from-women-positive-for-covid-19

\section{Hosted file}

Tabla 3.docx available at https://authorea.com/users/346819/articles/472667-umbilical-cordclamping-and-skin-to-skin-contact-in-deliveries-from-women-positive-for-covid-19 